\title{
COOPERAÇÃO COMO ESTRATÉGIA SEGUNDO DIFERENTES PERSPECTIVAS TEÓRICAS
}

\author{
Heron S. M. Begnis' \\ Eugênio Ávila Pedrozo \\ Vania de Fátima B. Estivalete ${ }^{3}$
}

\begin{abstract}
Resumo
Em um contexto no qual se evidencia a competitividade interorganizacional, este artigo procura identificar os enfoques centrais, os argumentos, as teorias de base e as principais conclusões alcançadas, ao final da década de 90 , sobre a temática da cooperação interorganizacional. O artigo orienta-se com base na revisão de literatura especializada e observa que os trabalhos científicos na área de estratégia, envolvendo cooperação, seguem três enfoques: a relação entre a utilização de estratégias cooperativas com o desempenho das firmas e/ou como fonte de vantagens competitivas; o estudo do processo de formação das configurações interorganizacionais de base cooperativa e a construção de modelos e frameworks enfatizando os aspectos teóricos subjacentes aos relacionamentos interorganizacionais. Nesse sentido, este artigo também identifica as abordagens teóricas utilizadas nos trabalhos sobre este campo da gestão estratégica e dos relacionamentos interorganizacionais cooperativos.
\end{abstract}

Palavras-chave: Relacionamentos interorganizacionais. Estratégia. Cooperação.

\section{INTRODUÇÃO}

A competição ainda domina o comportamento das firmas e não só representa o combustível para a prática da gestão estratégica como também estimula a maioria dos

\footnotetext{
' Doutorando em Agronegócios pela Universidade Federal do Rio Grande do Sul - CEPAN/UFRGS. Professor da Universidade de Santa Cruz do Sul - UNISC. Endereço: Av. Independência, 2293, Bairro Universitário, Santa Cruz do Sul, RS. CEP: 968I5-900. E-mail: heron@unisc.br

${ }^{2}$ Doutor em Administração pela Universidade Federal do Rio Grande do Sul - CEPAN/UFRGS. Professor e Pesquisador do Centro de Estudos e Pesquisas no Agronegócio da Universidade Federal do Rio Grande do Sul - CEPAN / UFRGS e do Programa de Pósgraduação em Administração - PPGA/UFRGS. Endereço: Av. João Pessoa, 31, Bairro Centro, Porto Alegre, RS. CEP: 90040-000. E-mail: eapedrozo@ea.ufrgs.br

${ }^{3}$ Doutoranda Em Agronegócios Pela Universidade Federal do Rio Grande Do Sul - CEPAN/UFRGS. Professora da Universidade Federal de Santa Maria - UFSM. Endereço: Av. Dois De Novembro, I377/60 I, Bairro Patronato, Santa Maria, RS. Cep: 97020-230. E-mail: vainafbe@terra.com.br Artigo recebido em: 20/03/2006. Aceito em: 28/02/2007. Membro do Corpo Editorial Científico responsável pelo processo editorial: Rolando Juan Soliz Estrada.
} 
ensaios acadêmicos nesse campo. Competir significava lutar contra adversários que deveriam ser derrotados ou eliminados. Mesmo quando se considerava a relação com fornecedores e clientes, a lógica que prevalecia era a do ganha-perde, sendo a competição um jogo de soma-zero, e, algumas vezes, até mesmo de soma negativa.

Enquanto se colocava em prática essa visão de competição, os mercados se concentravam e o ambiente no qual as organizações empresariais estavam inseridas se tornava cada vez mais inóspito e instável. Os riscos associados aos novos investimentos assumiam uma trajetória crescente, juntamente com a instabilidade gerada pelo rápido desenvolvimento tecnológico, cuja base se coloca na difícil e onerosa tarefa de gerar conhecimentos cada vez mais específicos. O ambiente complexo que se forma segundo estas características passa a requerer uma forma diferenciada de se pensar a competição. Mesmo as grandes corporações se tornaram pequenas frente ao desafio de continuar crescendo, ou, até mesmo de sobreviver sob essas mutantes condições dos mercados. A velha lógica competitiva não é mais a única regra, visto que novas formas de pensar e agir corporativamente se mostram como desafios estratégicos para as empresas. E é nesse contexto que se passa a olhar a competição sob uma ótica que busca reforço nas ações de cooperação. Os antigos rivais do mercado passam a ser vistos como potenciais parceiros e a estratégia da competição agora busca reforço nas ações de cooperação. Embora a lógica da cooperação esteja hoje na pauta dos estudos organizacionais, já na década de 30 se falava em cooperação e Brandenburg e Nalebuff (1996) foram reconhecidamente os pioneiros no uso do termo coopetição.

Child e Faulkner (1998), na tentativa de isolarem os principais motivos para a constituição de estratégias cooperativas, destacam três fases no processo econômico e industrial que podem ser identificados desde o final da II Guerra Mundial. Conforme esses autores, a primeira fase teve início logo depois da II Grande Guerra e caracterizou-se pela rigidez e proteção às economias estagnadas. A segunda fase, correspondeu aos anos 50 que marcaram a recuperação das economias nacionais afetadas pelo conflito armado, quando houve então o crescimento das empresas multinacionais: este período ficou destacado pela onda das organizações multidivisionais. Somente na terceira fase, a partir dos anos 80, marcada pelo crescimento de fundos de capital de risco, é que houve um forte crescimento de alianças estratégicas, principalmente nas áreas tecnológicas e de marketing. Além disso, conforme demonstra a Figura 1, ganharam importância as abordagens sobre cadeias de produção (MORVAN, 1985); cadeias de suprimento (BOWERSOX; CLOSS, 1996); joint ventures $(\mathrm{KOH}$; JEONGSUK; VENKATRAMAN, 1991; INKPEN; BEAMISH, 1997; PEARCE, 1997); alianças (OSBORN; HAGEDOORN, 1997; DAS; TENG, 1999; GULATI, 1998); supply chain management (COOPER et al., 1997); clusters (CRAVENS; PIERCY, 1994) e redes (PERROW, 1992; GRANDORI; SODA, 1995). 


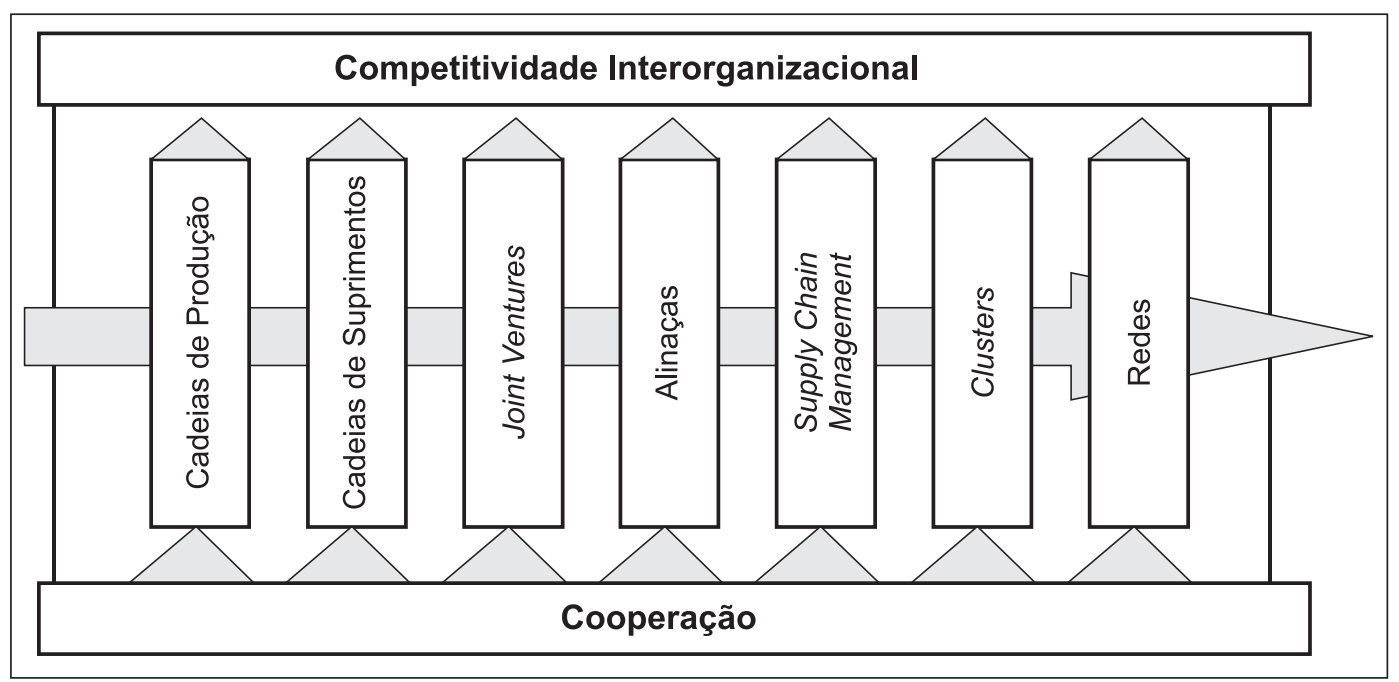

Figura 1 - A cooperação como base para a competitividade interorganizacional.

Fonte: Elaborado pelos autores.

Tais arranjos interorganizacionais têm a sua origem nos relacionamentos interfirmas, cuja base é a cooperação que envolve colaboração e parceria, e, o objetivo final é alcançar níveis diferenciados de competitividade: a competitividade interorganizacional. Frente a este contexto, o meio acadêmico respondeu inicialmente com vários trabalhos destacando as vantagens dos diferentes tipos de relacionamentos interorganizacionais cooperativos.

Nesse sentido, o presente trabalho parte da constatação de que, nos artigos publicados nos periódicos analisados, os arranjos cooperativos interfirmas avançaram, ao final dos anos 80 e início da década de 90 , juntamente com a pesquisa acadêmica desenvolvida pela área de gestão estratégica sobre o tema de cooperação. Assim, seguindo o pressuposto de que tais estudos tenham se consolidado no final dos anos 90 sobre as bases construídas no período anterior, esta revisão de literatura centrada na produção de língua inglesa caracteriza-se por ser um estudo prospectivo da forma como a cooperação interorganizacional tem sido abordada. Ou seja, procura identificar quais são os enfoques centrais; os principais argumentos empregados; as teorias de base e as principais conclusões dos artigos científicos do final da década de 90 que abordaram a temática da cooperação interorganizacional publicados nos três mais importantes periódicos internacionais de língua inglesa especializados nesse campo. Portanto, o artigo traz uma visão das produções sobre o tema da cooperação e não uma análise específica do fenômeno da cooperação interorganizacional.

Esse trabalho se orienta com base na revisão de literatura especializada e aponta que os artigos científicos na área da estratégia de cooperação seguem três tipos de 
enfoques: a relação entre a utilização de estratégias que envolvam a cooperação e o desempenho das firmas, ou seja, a cooperação como fonte de vantagens competitivas; o estudo do processo de formação dos arranjos interorganizacionais cooperativos e a construção de modelos e frameworks enfatizando os aspectos teóricos subjacentes aos relacionamentos interorganizacionais. Dentro desse objetivo central o artigo se estrutura, além desta introdução, em outras três partes. Na primeira parte, são evidenciados os aspectos metodológicos empregados para o seu desenvolvimento. Em seguida é apresentada uma visão panorâmica da produção acadêmica sobre cooperação como elemento estratégico na década de 90, enfatizando-se os últimos anos desse período. Por fim, são apresentadas as principais conclusões derivadas da análise dos artigos publicados pelos principais periódicos internacionais do campo da estratégia.

\section{METODOLOGIA}

Face ao objetivo proposto por esse trabalho, adotou-se a metodologia da pesquisa bibliográfica. De acordo com Gil (1991), a pesquisa bibliográfica é a que se desenvolve a partir de material já elaborado, tal como livros e artigos científicos, e particularmente aplicável em estudos exploratórios ou à análise das diferentes posições acerca de um determinado tema. A vantagem de uma pesquisa bibliográfica reside na possibilidade do investigador cobrir uma amplitude maior do fenômeno em consideração do que seria possível se fosse pesquisar diretamente (GIL, 1991).

No entanto, é necessário destacar que a metodologia da pesquisa bibliográfica apresenta a limitação decorrente da amplitude e qualidade das fontes de consulta. Nesse artigo, essa limitação também decorre da escolha da base de consulta restrita apenas a três periódicos de língua inglesa; porém, específicos e reconhecidamente importantes como veículo de divulgação dos avanços acadêmicos na área de estratégia e gestão. Também é importante salientar que esse artigo não tem o objetivo de esgotar o tema; mas de servir como referência para estudos mais avançados sobre o tema.

Seguindo a metodologia da pesquisa bibliográfica, esse artigo foi construído obedecendo às fases descritas por Gil (1991): identificação das fontes; localização das fontes e obtenção do material; leitura analítica do material; tomada de apontamentos; confecção de fichas e redação final. Deste modo, a primeira etapa correspondeu à definição das fontes de consulta. Como já mencionado, foram selecionados três periódicos internacionais de língua inglesa da área de estratégia e gestão que possuíam maior representatividade internacional e reconhecida excelência acadêmica. Assim, determinou-se que a base referencial para o estudo seria constituída pelo Strategic Management Journal; pela Academy of Management Journal e pela The Academy of Management Review.

O Strategic Management Journal (de periodicidade mensal) é um periódico que publica material original e inédito sobre todos os aspectos de interesse da adminis- 
tração estratégica. Ele é dedicado à melhoria e desenvolvimento da teoria e prática de administração estratégica e é projetado para atrair tanto gerentes práticos como acadêmicos teóricos. O Strategic Management Journal pretende representar um foro de comunicação para os avanços da teoria e prática da administração estratégica. Esse periódico possui como eixo central a estratégia e tem como tópicos principais os estudos sobre a alocação de recursos estratégicos; estrutura organizacional; liderança; empreendedorismo; métodos e técnicas para se avaliar e entender ambientes competitivos, tecnológicos, sociais e políticos; e processos estratégicos de decisão.

O The Academy of Management Journal (de periodicidade mensal) e o The Academy of Management Review (de periodicidade trimestral) são publicações da Academy of Management, uma associação de pesquisadores dedicada a criar e disseminar conhecimento sobre gestão e organizações. Essa associação foi fundada em 1963 e é hoje a maior e mais antiga associação mundial de pesquisa sobre gestão, contando com mais de 13 mil membros dispersos em 92 países. Esses dois periódicos estão entre os dez mais influentes e freqüentemente citados na área de gestão. Especialmente o The Academy of Management Review foi desenvolvido como periódico voltado à publicação de trabalhos teóricos de alta qualidade e mantém como política editorial a preocupação de promover novas perspectivas teóricas. Os artigos constantes nas diversas edições dos periódicos consultados foram obtidos, por meio eletrônico, através do ProQuest ${ }^{\circledR}$ e do Science Direct acessados a partir do Portal de Periódicos CAPES (Coordenação de Aperfeiçoamento de Pessoal de Nível Superior do Ministério da Educação).

A seleção dos artigos que passaram a compor este estudo efetivou-se com base na existência da evidente relação com os conceitos-chave que seriam trabalhados: alianças; joint ventures; redes; clusters; estratégias cooperativas; cooperação e relacionamentos interorganizacionais. Foram assim selecionados e trabalhados 35 artigos publicados no Strategic Management Journal, 22 artigos no Academy of Management Journal e 12 artigos publicados no The Academy of Management Review, totalizando 69 referências bibliográficas do período compreendido entre 1990 e 2000. Porém, destaca-se que a análise concentra-se nas publicações do final da década de 90, mais especificamente entre os anos de 1995 e 2000, quando, por suposição inicial desse estudo, considerou-se que já se poderia falar na consolidação da pesquisa sobre relacionamentos interorganizacionais cooperativos. Esta tarefa teve como eixo central o esclarecimento do que consistem as estratégias de cooperação e como elas podem ser analisadas segundo a perspectiva de diferentes autores. Após a seleção dos artigos, processou-se a leitura analítica do material selecionado, seguindo os objetivos de identificar as informações e estabelecer uma relação entre essas informações e o objetivo central proposto neste artigo. O procedimento de leitura analítica busca identificar, ordenar e sumariar as principais contribuições trazidas pelos diferentes autores. Esta etapa do trabalho, conforme os objetivos propostos, 
obedeceu aos seguintes passos: leitura dos textos selecionados; identificação das idéias-chave trabalhadas em cada artigo; organização destas idéias segundo as diferentes perspectivas adotadas pelos autores; e, finalmente, a síntese dos conceitos e a elaboração do texto final do artigo.

\section{ANTECEDENTES: OS TRABALHOS SOBRE ARRANJOS COOPERATIVOS INTERFIRMAS NO FINAL DOS ANOS 80 E INÍCIO DA DÉCADA DE 90}

\subsection{As contribuições do Strategic Management Journal}

As publicações sobre estratégias de cooperação encontradas no Strategic Management Journal nos primeiros anos da década de 90 são marcadas pela apresentação do tema cooperação como nova forma de arranjo interorganizacional voltado à melhoria do desempenho das empresas. Muitos dos trabalhos desse período foram, de alguma forma, influenciados pelo artigo de Nielsen (1988), que procurava demonstrar que as estratégias cooperativas poderiam melhorar a eficiência das organizações de modo ético em várias circunstâncias. Nielsen (1988), utilizou uma abordagem multidisciplinar baseada na gestão estratégica, na biologia evolucionária, na teoria dos jogos e na teoria dos ecossistemas para demonstrar a utilidade das estratégias cooperativas frente às noções de ciclo de vida ambiental; estratégias genéricas e de valor agregado. Concluiu que as estratégias de cooperação entre grandes corporações podem ser mais eficientes que os mecanismos externos do mercado, ou seja, que as estratégias cooperativas podem melhorar a eficiência das organizações em diferentes ambientes de mercado. Dentro da linha proposta por Nielsen (1988), cabe salientar, entre as publicações do Strategic Managemente Journal no período de 1990 a 1995, os artigos de: Ring e Van de Ven (1992), que trata da forma como se estruturam os relacionamentos cooperativos entre as organizações; o trabalho de Hagedoorn e Schakenraad (1994), dentre aqueles que procuram relacionar o desempenho das organizações com a adoção de estratégias que envolvem a cooperação; e o artigo de Mohr e Spekman (1994) sobre as características das parcerias de sucesso. Ring e Van de Ven (1992), com base na Economia dos Custos de Transação, procuram demonstrar quais são as forças que levam as organizações a buscar relações cooperativas com outras empresas e que mecanismos estão disponíveis para governar estes tipos de relações interorganizacionais.

A questão central de Hagedoorn e Schakenraad (1994) se coloca no sentido de explicar em qual medida as parcerias estratégicas tecnológicas interfirmas afetam a lucratividade das empresas comprometidas em tais esforços conjuntos. Discutir as características das parcerias que estão associadas com o sucesso também é o objetivo do artigo de Mohr e Spekman (1994), que se concentra nas relações diádicas verticais entre a manufatura e a distribuição na indústria de computadores pessoais. 
Estes mesmos autores concluíram que as variáveis mais significantes para a predição do sucesso de uma parceria são: a coordenação: o comprometimento: a confiança: a qualidade da comunicação: o compartilhamento de informações: a participação e a forma adotada para a solução de eventuais problemas que surgem da união.

\subsection{The Academy of Management Journal: o reforço da base empírica}

No início da década de 90, as publicações do The Academy of Management Journal sobre estratégias cooperativas estavam direcionadas principalmente para a análise do processo de formação e estruturação dos arranjos cooperativos. Nessa linha temática e dentre os trabalhos desse período, salienta-se o artigo de Heide e Miner (1992), sobre os efeitos das interações da freqüência dos contatos prévios entre parceiros comerciais para a efetivação das ações de cooperação. Os autores identificam quatro áreas com potencial interesse para a cooperação: flexibilidade: troca de informações: solução compartilhada de problemas e restrições ao uso do poder.

O artigo de Browning, Beyer e Shetler (1995), parte da Teoria da Complexidade para analisar a construção da cooperação em indústrias competitivas e traz uma interessante contribuição para o entendimento da formação das alianças. Segundo os autores, os fatores que antecedem a formação da cooperação são a ambigüidade e a desordem. Na medida em que se vai construindo uma moral comum entre os agentes e firmas envolvidos, sem que se espere um retorno individual e imediato, isso contribui para a consolidação da aliança.

A instabilidade presente nas alianças estratégicas já havia sido destacada por Parkhe (1993), que utilizou o referencial da Economia dos Custos de Transação e da Teoria dos Jogos para a análise da estruturação de alianças estratégicas. Conforme Parkhe (1993), algumas estruturas das alianças são mais propensas a fraudes, a comportamentos muito imprevisíveis e a fraca estabilidade, advertindo que a estrutura está diretamente ligada ao desempenho.

Esses trabalhos do início da década têm dado ênfase à importância da estrutura que se constrói com as ações de cooperação. Gulati (1995), colabora nesse sentido ao explorar os fatores que explicam a escolha das estruturas de governança nas alianças interorganizacionais, enfatizando os custos de transação. O autor conclui que a escolha das formas contratuais em alianças não depende somente das atividades incluídas na parceria e dos custos de transação associados a elas. Em vez disso, reforça Gulati (1995), a escolha das estruturas de governança depende da confiança que emerge ao longo do tempo entre as organizações e os seus repetitivos vínculos. Por fim, outros dois trabalhos desse período abordam o impacto das joint ventures sobre o valor de mercado das firmas (KOH; JEONGSUK; VENKATRAMAN, 1991; MADHAVAN; PRESCOTT, 1995). 


\subsection{The Academy of Management Review: o foco nos relacionamentos}

As primeiras publicações da The Academy of Management Review no início da década de 90 trazem, para o campo das estratégias cooperativas as contribuições de Hill (1990): Oliver (1990): Ghoshal e Bartlett (1990); e, Ring e Van de Ven (1994). O trabalho de Hill (1990), contribui no sentido de aprofundar o entendimento sobre o risco de comportamentos oportunísticos que envolve as relações interorganizacionais, não sendo muito específico em termos de estratégias de cooperação interfirma. Mas o artigo de Oliver (1990) traz uma visão mais ampla sobre os relacionamentos interfirmas.

Oliver (1990), ao trabalhar com os determinantes dos relacionamentos interorganizacionais destaca que a literatura deste campo tem consagrado seis determinantes gerais da formação de relacionamentos: necessidade; assimetria: reciprocidade: eficiência: estabilidade e legitimidade. No entanto, a contribuição central de Oliver (1990), está em mostrar que as contingências críticas de necessidade, assimetria, estabilidade e legitimidade são determinadas principalmente por fatores externos. Já as contingências de eficiência são influenciadas em grande parte por fatores internos e pelo custo que envolve a própria relação. Finalmente, as contingências de reciprocidade são principalmente afetadas pelas características relativas ou comparativas dos participantes e do grau de congruência que se apresenta entre eles.

Embora não exatamente tratando de relacionamentos cooperativos interorganizacionais, o artigo de Ghoshal e Bartlett (1990), se configura num exemplo de como o corpo teórico já desenvolvido sobre os relacionamentos interfirmas pode ser utilizado para estudos em outros campos da gestão. O objetivo do artigo de Ghoshal e Bartlett (1990), é propor uma formulação inicial considerando a forma como os conceitos e ferramentas das análises interorganizacionais podem ser aplicadas para se ajustar a um caso diferente; mas análogo, representado pelas empresas multinacionais. Talvez a principal contribuição trazida por The Academy of Management Review, ainda na primeira metade da década considerada, seja o artigo de Ring e Van de Ven (1994). Nesse trabalho os autores propõem um framework para a análise do desenvolvimento do processo de relacionamentos interorganizacionais cooperativos.

Ring e Van de Ven (1994), partem da suposição de que o processo, ou seja, a seqüência de eventos e interações entre as empresas, é a questão central quando se trata de relacionamentos interorganizacionais, pois o modo como os agentes negociam, executam e modificam os termos do relacionamento, influencia o grau com que os participantes julgam a relação justa e eficiente.

\section{A PRODUÇÃO ACADÊMICA SOBRE COOPERAÇÃO COMO ESTRATÉGIA NO FINAL DA DÉCADA DE 90}

O campo das estratégias cooperativas e dos relacionamentos interorganizacionais foi bastante fértil durante a década de 90 , quando foram produzidos vários trabalhos 
sobre o tema. Na seqüência deste estudo, é apresentada uma revisão sintética dos trabalhos que foram publicados no final desse período por três dos principais periódicos internacionais sobre gestão e estratégia.

\subsection{Os trabalhos do Strategic Management Journal: ênfase na formação dos relacionamentos}

Os artigos publicados no Strategic Management Journal no final da década de 90 passam a apresentar três linhas temáticas bem definidas e destacadas na Figura 02. Entre 1996 e 2000, os trabalhos publicados nesse periódico podem ser reunidos em três grupos. Num primeiro grupo reúnem-se os trabalhos cujo tema central é a análise do desempenho das firmas envolvidas em algum tipo de cooperação interfirmas, assim como, a investigação sobre as vantagens competitivas decorrentes dessas estratégias. Esses artigos sobre as vantagens das estratégias de cooperação e sua influência sobre o desempenho das firmas estão alicerçados em evidências empíricas, com exceção do texto de Gulati, Nohria e Zaheer (2000), que faz um comentário geral sobre diferentes artigos da edição especial sobre redes estratégicas desse periódico.

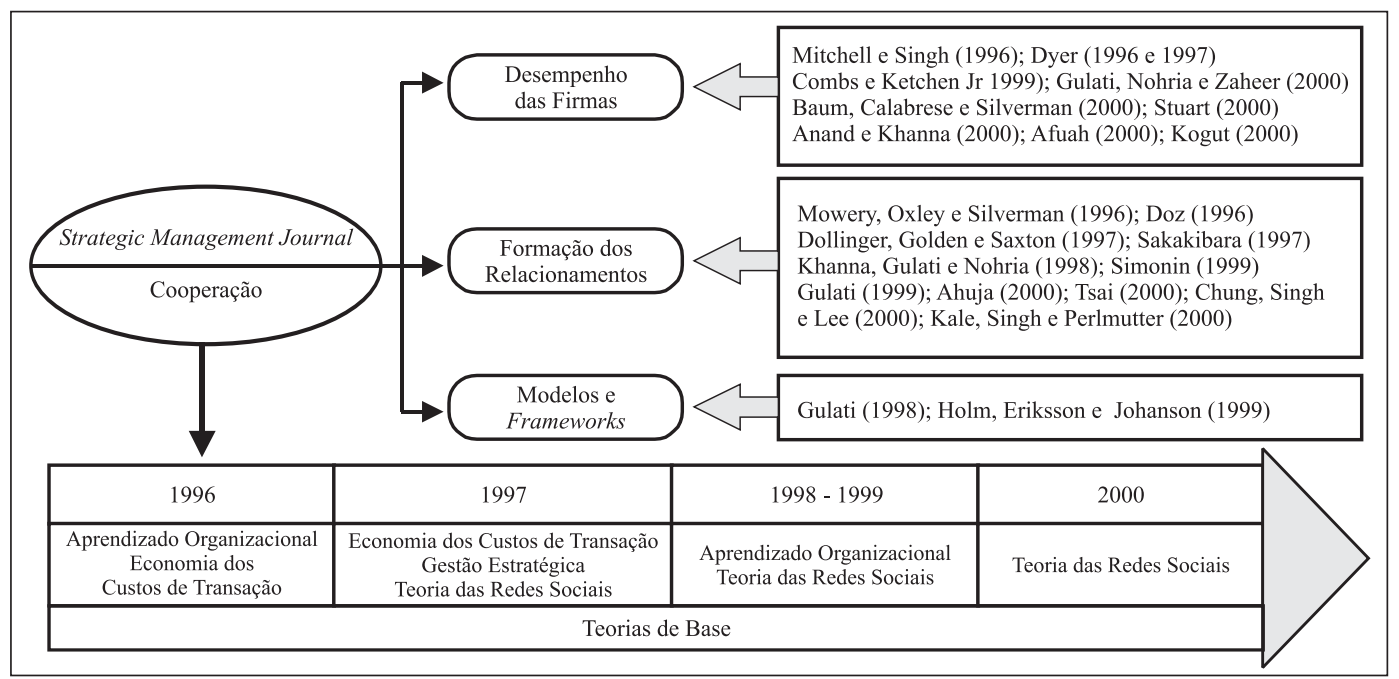

Figura 2 - Os diferentes temas e enfoques teóricos presentes nos artigos do Strategic Management Journal no final da década de 1990.

Fonte: Elaborado pelos autores.

O segundo grupo de trabalhos é composto por artigos que tratam dos motivos e condições necessárias ou pretendidas para que se adotem ações cooperativas. No período analisado, esse tema reúne em seu entorno o maior número de artigos publicados no Strategic Management Journal. Por fim, num terceiro grupo podem ser 
reunidos os artigos que procuram trazer alguma contribuição teórica para o campo da cooperação, propondo e testando modelos teóricos e frameworks.

\subsubsection{Vantagens competitivas e desempenho das firmas coopetitivas: a base empírica}

Uma das formas de se justificar o estabelecimento de relacionamentos colaborativos é percebê-los como estratégia de permanência nos negócios. Nesta linha, Mitchell e Singh (1996), com base numa perspectiva teórica gerencial, argumentam que os negócios que envolvem a comercialização de bens complexos se beneficiam quando as firmas colaboram mais proximamente. Nesse sentido, a colaboração é vista como uma forma de superar a limitação das competências individuais das firmas. Constatações semelhantes também foram encontradas por Dyer (1996 e 1997),ao analisar as redes de empresas da indústria automotiva americana e japonesa sob a ótica dos custos de transação. $\mathrm{O}$ autor concluiu que existe uma relação positiva entre especificidade dos ativos da cadeia de valor criada pela rede de empresas e o desempenho geral e cita que os fatores que influenciam a geração da vantagem competitiva por uma estratégia de rede estão relacionados ao ambiente institucional (custos de contrato, confiança); à incerteza e à volatilidade da indústria (choques externos) e à interdependência do produto/tarefa.

Após 1996, os estudos sobre vantagens competitivas e de desempenho das estratégias cooperativas somente retornam nas publicações do Strategic Management Journal no ano de 1999 com o artigo de Combs e Ketchen (1999), e em 2000 com os trabalhos de Baum, Calabrese e Silverman (2000); Anand e Khanna (2000); Afuah (2000) e Kogut (2000). Combs e Ketchen (1999), procuram explicar a cooperação interfirma e a performance destas organizações através da "Visão Baseada em Recursos e da Economia Industrial”. A Visão Baseada em Recursos sugere que as empresas busquem capitalizar e incrementar as suas capacidades e habilidades, enquanto que a Economia Industrial afirma que as empresas deveriam focalizar na minimização dos custos organizacionais.

Baum, Calabrese e Silverman (2000), encontram diferenças no desempenho inicial de empresas novas ao avaliarem a composição das redes e alianças na indústria canadense de biotecnologia, comprovando suas hipóteses de que as firmas iniciantes podem melhorar sua performance através do estabelecimento de alianças e da configuração destas alianças numa rede. Através de outra linha de trabalho, Kogut (2000), considerando que um dos critérios para se medir o desempenho de uma firma seja o seu valor de mercado, propõe que parte do valor de uma firma deriva da sua participação numa rede colaborativa. De forma complementar, coloca-se o estudo de Afuah (2000), cujas constatações reforçam o argumento de que, principalmente num 
ambiente tecnológico mutável, é necessário perceber que os recursos estratégicos residem na rede e não nas firmas individuais. Também, nesse sentido, Anand e Khanna (2000), argumentam que a importância do aprendizado sobre a criação de valor é um forte motivo para que se aprofunde a pesquisa sobre alianças, principalmente em situações caracterizadas por grandes ambigüidades contratuais.

\subsubsection{A construção dos arranjos cooperativos: desvendando os relacionamentos}

Sobre a construção dos arranjos cooperativos, o Strategic Management Journal, traz um conjunto de onze artigos tratando dessa temática. Destes, os trabalhos de Mowery, Oxley e Silverman (1996); Doz (1996); Dollinger, Golden e Saxton (1997); Sakakibara (1997); Ahuja (2000); Gulati (1999); Tsai (2000); Chung, Singh e Lee (2000); Simonin (1999); Kale, Singh e Perlmutter (2000), trazem evidências empíricas sobre suas proposições. Nessa temática de formação dos arranjos cooperativos, apenas o trabalho de Khanna, Gulati e Nohria (1998,) trata a questão apenas sob o prisma teórico.

O artigo de Doz (1996), explora a evolução da cooperação através de alianças estratégicas entre firmas, utilizando o enfoque da Teoria Organizacional e abordando a questão do Aprendizado Organizacional, tendo por objetivo avaliar a contribuição relativa das condições iniciais e do processo evolucionário do aprendizado nos resultados de alianças estratégicas. A maior parte dos textos que trata da formação de arranjos interorganizacionais cooperativos enfatiza o papel da reputação dos parceiros sobre a decisão de se implementar uma estratégia desse tipo. Sobre a questão da reputação, o artigo de Dollinger, Golden e Saxton (1997), indica que a reputação é um construto multidimensional que as características pessoais dos tomadores de decisão podem se sobrepor à própria reputação da firma e que a reputação é um elemento que afeta as decisões independentemente do objetivo pretendido ser uma aliança com um fornecedor ou com um competidor. Nesse sentido, a confiança derivada da reputação dos parceiros, não só se apresenta como fator de sucesso das estratégias cooperativas; mas também é elemento chave do processo de sua formação.

Quanto aos motivos que levam ao estabelecimento de parcerias pode-se identificar, nos diferentes trabalhos que abordam esta linha de investigação, uma convergência em torno de alguns objetivos principais. Um exemplo é o artigo de Sakakibara (1997), que apresenta a proposição de que a heterogeneidade das capacitações dos participantes de consórcios de $P \& D$ representa uma condição para distinguir dois motivos para a cooperação: o compartilhamento de custos e o compartilhamento de habilidades.

Gulati (1999), desenvolve um estudo dinâmico do papel dos recursos da rede na determinação da formação das alianças. Os resultados desse estudo confirmam as observações de que recursos que são acumulados pelas redes surgem da própria participação das firmas nas redes e das suas experiências acumuladas em alianças anteriores. 
O autor ainda apresenta o argumento de que esta experiência acumulada pelas firmas, em virtude da participação anterior em outras redes, irá influenciar a decisão destas em entrar em novas redes. Ainda dentro dessa abordagem das redes sociais, destacase o artigo de Tsai (2000), que trata da dinâmica evolucionária da formação de redes através da análise de como as unidades organizacionais criam novas ligações interunidades para a troca de recursos. Por fim, o trabalho de Chung, Singh e Lee (2000), praticamente oferece uma conexão entre os temas abordados por Sakakibara (1997), Ahuja (2000) e Tsai (2000), ao afirmar que a base sobre a qual se formam as alianças entre as organizações depende da complementaridade dos recursos, da similaridade do status das organizações envolvidas e do capital social. Chung, Singh e Lee (2000), acrescentam que o capital social surge das experiências colaborativas diretas e indiretas das organizações, e assume um importante papel na formação das alianças.

Kale, Singh e Perlmutter (2000), também consideram que uma das maiores razões para que as firmas participem de alianças é adquirir know-how e capacidades dos seus parceiros de aliança. Mas, ao mesmo tempo, as firmas buscam se proteger do comportamento oportunista dos seus parceiros, retendo seus próprios ativos essenciais. Destaca-se aqui, que a pesquisa sobre estratégias cooperativas avança com base no campo da Teoria das Redes Sociais e na importância atribuída ao capital social ou relacional.

\subsubsection{Teoria da estratégia de cooperação: frameworks e modelos}

Alguns trabalhos publicados no Strategic Management Journal no final da década de 90 assumem a tentativa de construir uma "teoria da estratégia cooperativa". Dentre estes, podem ser destacados os artigos de Gulati (1998), que trazem uma agenda de pesquisa para a área e o trabalho de Holm, Eriksson e Johanson (1999), no qual propõe e testam um modelo estrutural de relacionamentos de negócios. Gulati (1998), por exemplo, introduz a perspectiva das redes sociais para o estudo das alianças estratégicas, motivado pela perspectiva de que a formação e os resultados das alianças podem ser definidos e formados pelas redes sociais nas quais muitas firmas estão imbricadas. $\mathrm{O}$ autor identifica cinco assuntos-chave que deveriam fazer parte de uma agenda de pesquisa sobre alianças: (a) a formação das alianças; (b) a escolha das estruturas de governança; (c) a dinâmica evolutiva das alianças; (d) a performance das alianças e (e) os motivos pelos quais as firmas entram numa aliança.

Holm, Eriksson e Johanson (1999), desenvolvem e testam um modelo estrutural de relacionamentos cujo objetivo é investigar a relação entre a interação, interdependência e a criação de valor nas relações de negócios em redes. Estas relações são conceituadas numa cadeia casual de conexão de negócios em redes através de um compromisso mútuo para a dependência mútua e a relação de criação de valor. Os resultados sobre a cadeia causal investigada por Holm, Eriksson e Johanson (1999), demonstram que o desenvolvimento da relação tem um efeito forte na criação de seu valor. 


\subsection{Artigos do The Academy Management Journal: foco no desempenho}

Ao se deter na análise dos artigos publicados no The Academy of Management Journal no final da década de 90, percebe-se o predomínio de trabalhos enfatizando a avaliação dos resultados das firmas envolvidas em ações de cooperação (Figura 3). Apenas o artigo de Osborn e Hagedoorn (1997), procura se aprofundar somente nos aspectos teóricos envolvidos nas estratégias de cooperação.

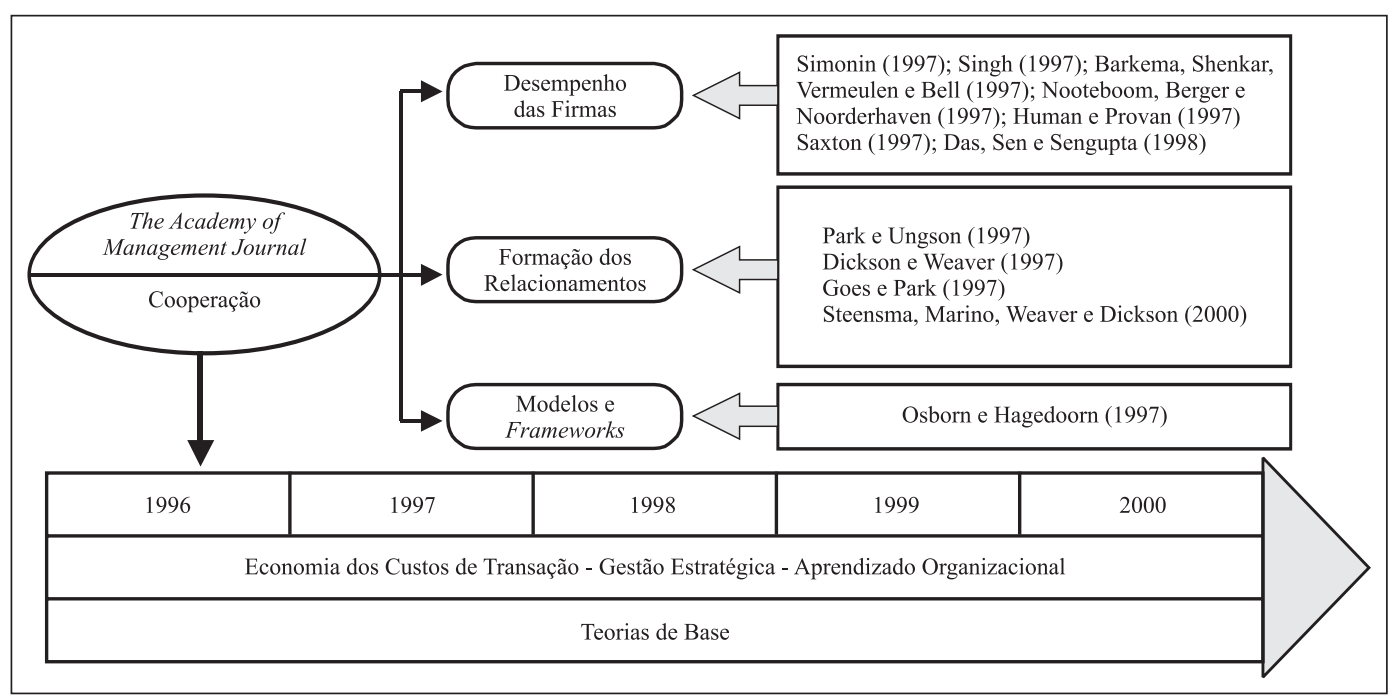

Figura 3 - Os diferentes temas e enfoques teóricos presentes nos artigos do The Academy of Management Journal no final da década de 90.

Fonte: Elaborado pelos autores.

Osborn e Hagedoorn (1997), trabalham com a institucionalização e a dinâmica evolucionária das alianças interorganizacionais e das redes como base para a construção de uma teoria integrada sobre redes e alianças interorganizacionais. Os autores advertem que o problema das alianças reside na escolha dos parceiros cujas estratégias se assemelham aos objetivos da firma em particular. Também consideram que cada participante de uma rede pode ter uma clara missão dentro desta, porém as intenções dos participantes em cada tipo de aliança ou rede podem ser amplamente diferentes.

O The Academy of Management Journal, no final da década de 90, apresenta quatro artigos substanciados com evidências empíricas que tratam da formação das redes e alianças cooperativas. Os trabalhos de Park e Ungson (1997); Dickson e Weaver (1997); Goes e Park (1997); Steensma et al. (2000), seguem exatamente essa linha de investigação. Park e Ungson (1997), examinam os efeitos da nacionalidade dos parceiros, as diferenças organizacionais e a motivação econômica sobre a dis- 
solução das joint ventures e concluem que as diferenças culturais não implicam na dissolução das parcerias porque os relacionamentos que antecedem a sua formação trabalham no sentido de negar algumas das complexidades que surgem a partir das diferenças culturais. Não obstante, as ameaças vindas do comportamento oportunista e da rivalidade são indicadores mais fortes sobre a dissolução das joint ventures do que as variáveis puramente organizacionais.

Partindo do mesmo ponto que Park e Ungson (1997), Steensma et al. (2000), também analisam os efeitos das culturas nacionais sobre a possibilidade de formação de alianças tecnológicas e chegam a conclusões não muito semelhantes as de Park e Ungson (1997). As constatações de Steensma et al. (2000), indicam que a força das culturas nacionais influenciam diretamente a formação de alianças tecnológicas e se colocam como moderadoras da relação entre a incerteza tecnológica percebida e a formação das alianças. Especificamente, os autores sugerem que a explicação advinda da dependência de recursos para a formação de alianças tecnológicas é muito forte quando se consideram sociedades que cultuam valores cooperativos e procuram evitar a incerteza.

De outro lado, a explicação para a formação de alianças dada pela Economia dos Custos de Transação somente é válida em sociedades que valorizam o individualismo. Esses dois estudos concentram os motivos para a formação das alianças muito mais em aspectos externos às próprias firmas envolvidas. Os aspectos ambientais e organizacionais envolvidos no processo de decisão sobre a formação de alianças são considerados no artigo de Dickson e Weaver (1997). Estes autores reforçam a idéia de multidimensionalidade da incerteza ambiental, afirmando que a forma como os gestores percebem este tipo de incerteza se mostra como um fator determinante sobre a decisão de se utilizar alianças.

O aprendizado através dos relacionamentos interorganizacionais é um dos motivos mais freqüentemente citados nos trabalhos sobre as razões que levam as firmas a adotarem estratégias cooperativas. Por conseqüência, deve existir uma forte ligação entre o aprendizado e o desempenho obtido pelas firmas envolvidas em arranjos interorganizacionais cooperativos. Envolvendo como tema central a relação entre cooperação e desempenho organizacional, o The Academy of Management Journal, no final da década de 90 traz os artigos de Simonin (1999); Barkema, Shenkar, Vermeulen e Bell (1997); Singh (1997); Nooteboom, Berger e Noorderhaven (1997); Human e Provan (1997); Saxton (1997) e Das, Sen e Sengupta (1998), todos procurando demonstrar empiricamente suas proposições.

\subsection{The Academy of Management Review: a ECT como base teórica}

Na metade final da década de 90, as publicações trazidas pelo The Academy of Management Review (destacadas na Figura 4), basicamente têm como foco os 
aspectos teóricos dos relacionamentos cooperativos interorganizacionais através dos trabalhos de Lado, Boyd e Hanlon (1997); Inkpen e Beamish (1997) e Doney, Cannon e Mullen (1998).

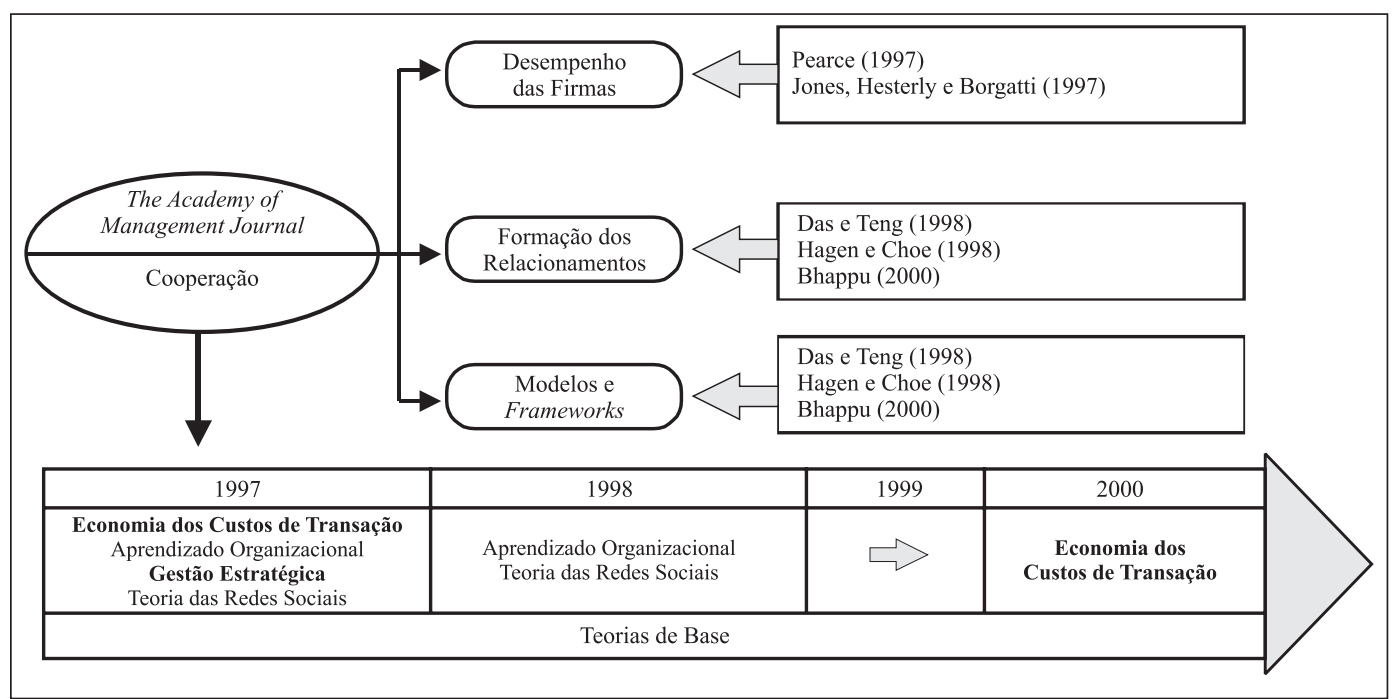

Figura 4 - Os diferentes temas e enfoques teóricos presentes nos artigos do The Academy of Management Review no final da década de 90.

Fonte: Elaborado pelos autores.

Lado, Boyd e Hanlon (1997), propõem a noção de comportamento do tipo rentseeking (tipo de conduta dos agentes econômicos "caçadores" de renda) para explicar como as firmas podem gerar rendas econômicas e alcançar um desempenho superior duradouro, simultaneamente, através da competição e da cooperação. Entendendo competição e colaboração como dimensões distintas; mas inter-relacionadas, os autores desenvolvem um framework que apresenta uma tipologia de quatro células representando tipos diferentes de comportamento rent-seeking. As proposições de Lado, Boyd e Hanlon (1997), fazem uma ligação entre comportamentos do tipo rent-seeking, competências organizacionais e desempenho dos negócios, com o objetivo de guiar os futuros esforços de pesquisa. E nesse sentido, destacam a força e a utilidade das teorias das ciências sociais.

Inkpen e Beamish (1997), desenvolvem um framework para análise da instabilidade das joint ventures internacionais, baseado no poder de barganha e na perspectiva da dependência. Para estes autores, a instabilidade representa o maior desafio na relação entre os parceiros, sendo que, o seu principal argumento desenvolvido é de que a instabilidade das joint ventures internacionais está associada às modificações do poder de barganha dos parceiros. Expandindo a idéia desenvolvida por Inkpen e 
Beamish (1997), pode-se estabelecer uma associação entre a instabilidade decorrente da variação do poder de barganha dos parceiros e a dificuldade de se estabelecer níveis consideráveis de confiança. Sobre este tema da confiança, Doney, Cannon e Mullen (1998), desenvolvem um framework identificando cinco processos cognitivos baseados na confiança e que explicam como esta se desenvolve no contexto dos negócios. A noção de confiabilidade entre os parceiros de uma aliança é também examinada teoricamente por Das e Teng (1998), entre os artigos do The Academy of Management Review sobre a formação dos relacionamentos. Estes autores sugerem que a confiabilidade deriva de duas fontes distintas: confiança e controle. Segundo esses autores, para que uma aliança estratégica tenha sucesso é fundamental que exista confiança na cooperação dos parceiros, sendo esta definida por eles como a certeza percebida pela firma sobre a cooperação satisfatória do parceiro.

Confiança é igualmente o elemento-chave do artigo de Hagen e Choe (1998) e Bhappu (2000), ambos trazendo evidências empíricas baseadas na cultura japonesa. Hagen e Choe (1998), apresentam a confiança como a força que conduz as relações interorganizacionais entre as firmas japonesas. A abordagem apresentada por Hagen e Choe (1998), se mostra interessante porque ao invés de considerarem a confiança como uma força inerente que conduz os relacionamentos interfirmas no Japão, propõem que a combinação de mecanismos institucionais e de sansão social é largamente responsável pela aparente cooperação induzida pela confiança. Por fim, a análise dos trabalhos sobre estratégias cooperativas publicados pelo The Academy of Management Review no final dos anos 90, destaca os artigos teóricos de Pearce (1997) e Jones, Hesterly e Borgatti (1997), sobre a relação entre relacionamentos interorganizacionais e desempenho das firmas. Pearce (1997), procura explicar o desempenho das joint ventures a partir da abordagem da Economia dos Custos de Transação. Este autor argumenta que a estrutura de governança representada pelas joint ventures impõe onerosos custos de barganha e influência política às firmas envolvidas neste tipo de arranjo cooperativo interorganizacional. Pearce (1997), então conclui que esses custos de transação afetam negativamente a adaptação organizacional, a performance e a sobrevivência das firmas que adotam a estratégia de formação de joint ventures.

\section{PRINCIPAIS ELEMENTOS CONSIDERADOS SOBRE A COOPERAÇÃO COMO ESTRATÉGIA}

O objetivo central do presente estudo é determinar o "estado da arte" no campo das estratégias envolvendo a cooperação interorganizacional: identificando quais são os enfoques centrais: os principais argumentos empregados: as teorias de base e as principais conclusões a que chegaram os autores que trabalharam sobre nesse 
tema no final da década de 90 . Dentro desse objetivo, a análise que se procedeu com base na revisão de literatura especializada, resultou na percepção de que os trabalhos científicos na área da estratégia de cooperação seguem três tipos de enfoques: a relação entre a utilização de estratégias cooperativas e desempenho das firmas ou como fonte de vantagens competitivas (Grupo A); o estudo do processo de formação dos arranjos interorganizacionais cooperativos (Grupo B) e a construção de modelos e frameworks enfatizando os aspectos teóricos que envolvem os relacionamentos interorganizacionais (Grupo C).

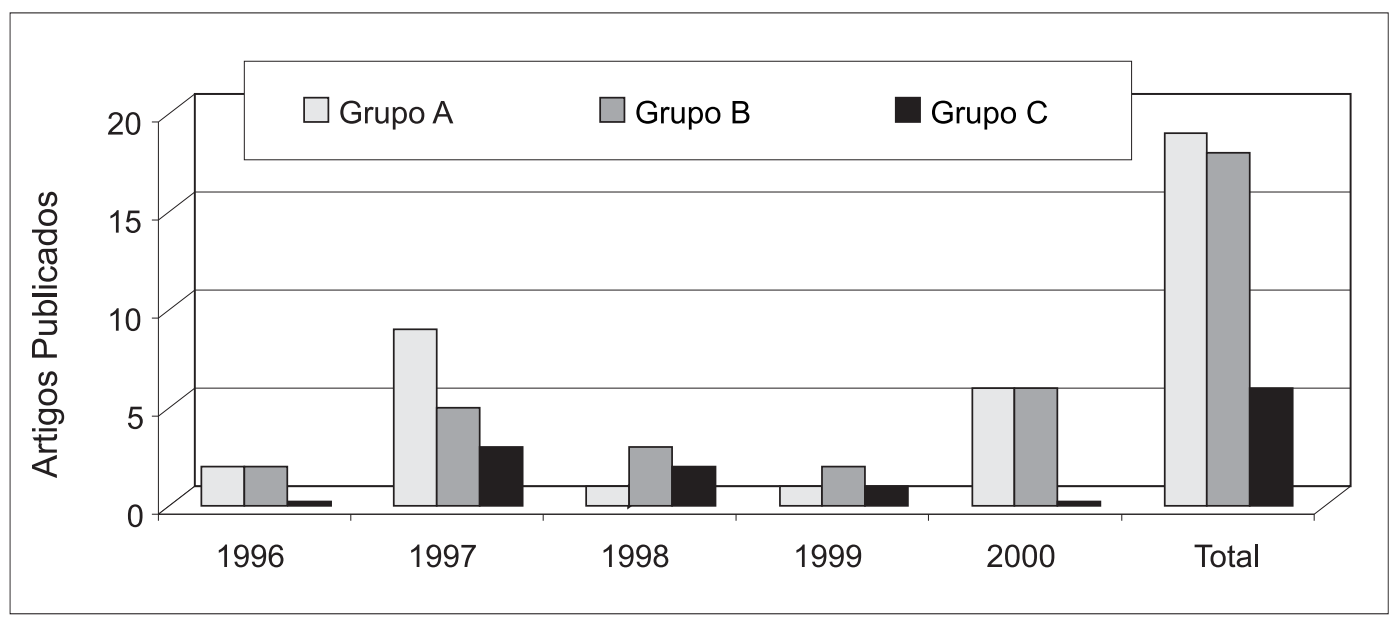

Gráfico 1 - Número de artigos publicados entre 1996 e 2000 nos periódicos analisados.

Fonte: Elaborado pelos autores.

De forma geral, como se visualiza no Gráfico 1, a análise mostrou que existe a predominância de artigos voltados à análise do desempenho das firmas envolvidas em relacionamentos cooperativos interorganizacionais (Grupo A). Nesse sentido, os trabalhos têm constatado que as firmas envolvidas de alguma forma em arranjos cooperativos, melhoram seus desempenhos frente a outros tipos de relacionamentos, tal como as relações típicas de mercado. Segundo a maior parte dos autores revisados, esta melhoria na performance das empresas se mostra mais evidente quando a cooperação envolve pesquisa e desenvolvimento, principalmente no caso de tecnologias complexas.

Sobre os estudos que tratam do processo de formação dos arranjos interorganizacionais cooperativos (Grupo B), pode-se concluir que estes tratam a confiança, o aprendizado e o compartilhamento de recursos e habilidades como os elementos centrais, tanto para formação como para o sucesso desses tipos de relacionamentos. 
A ênfase dos trabalhos nesse campo recai sobre o processo de seleção dos parceiros para a colaboração, colocando como fatores-chave deste processo de estabelecimento de arranjos cooperativos interorganizacionais a reputação, a confiança, o oportunismo e a formação de capital social. Destaca-se, em complemento, o pequeno número de trabalhos objetivando avançar sobre as questões teóricas que envolvem os relacionamentos interorganizacionais (Grupo C). Não obstante, deve-se destacar a relevância acadêmica dessa linha de trabalho, o que indica a necessidade de se avançar sobre a construção de um corpo teórico mais robusto sobre cooperação e estratégias interorganizacionais.

Por fim, ao se considerar as abordagens teóricas empregadas nos artigos analisados percebe-se uma relativa pluralidade dessas abordagens. No início do período analisado há predominância das Teorias Organizacionais e principalmente da utilização do enfoque do Aprendizado Organizacional. Nos anos de 1997 e 1998, cresce significativamente o número de trabalhos que utilizam a abordagem da Economia dos Custos de Transação, que representa o enfoque teórico mais utilizado para a sustentação das análises pretendidas pelos diversos autores durante o período analisado.

A partir de 1997, um conjunto maior de abordagens teóricas passa a ser utilizado agregando às mais freqüentemente utilizadas: a Visão Baseada em Recursos e a Gestão Estratégica; outras abordagens como o enfoque da Organização Industrial; a Teoria da Agência; a Teoria da Dependência de Recursos e a Teoria dos Jogos; mas principalmente destaca-se que a partir de 1997 os estudos sobre os relacionamentos cooperativos interorganizacionais têm avançado com o auxílio do novo enfoque trazido pela Teoria das Redes Sociais, especialmente tratando da formação de capital social como base para a formação de alianças estratégicas.

\section{CONSIDERAÇÕES FINAIS}

Frente ao objetivo central de identificar os enfoques e as teorias de base sobre a temática da cooperação interorganizacional, acredita-se que esse trabalho tenha colaborado no sentido de mostrar quais são as principais contribuições no campo da cooperação como estratégia, fornecendo uma visão ampla das principais questões que envolvem a formação e o desempenho dos diferentes arranjos cooperativos interorganizacionais. Em síntese, os textos analisados apontam para vantagens em termos de redução de custos das transações dos arranjos cooperativos frente aos relacionamentos competitivos interfirmas e que a confiança e o aprendizado são elementos centrais, tanto para formação como para o sucesso destes tipos de relacionamentos. Em termos de abordagens teóricas utilizadas, há um predomínio da Economia dos Custos de Transação, das Teorias Organizacionais de modo geral e, dentro destas e em especial, do ponto de vista do Aprendizado Organiza- 
cional. Cabe ainda destacar que os estudos sobre relacionamentos cooperativos interorganizacionais têm avançado com o auxílio de novos enfoques trazidos pela Teoria das Redes Sociais, especialmente ao tratar a formação de capital social como base para a formação de alianças estratégicas. E, nessa direção, há um vasto espaço para a discussão sobre o papel da confiança na base dos relacionamentos interorganizacionais cooperativos.

Por fim, acredita-se que este trabalho trouxe um novo aporte ao campo da estratégia interorganizacional, no sentido de mostrar quais são as suas abordagens teóricas mais utilizadas, servindo como subsídio para novas pesquisas dentro dessa área. Ao final deste trabalho, evidencia-se a possibilidade de se falar em uma nova tipologia de estratégia: a Estratégia Cooperativa, tal como já havia sido indicado por Nielsen (1988), no próprio título de seu artigo (Cooperative Strategy) publicado pelo Strategic Management Journal no final da década de 80. Partindo da mesma base utilizada nesta pesquisa, um novo trabalho poderia verificar em que sentido cada uma destas diferentes correntes teóricas estaõ sendo empregadas e quais as suas contribuições específicas para o estudo dos relacionamentos cooperativos interorganizacionais. Também, sob a perspectiva das redes sociais, novos trabalhos poderiam investigar o papel dos agentes e dos relacionamentos interpessoais na formação de arranjos cooperativos interfirmas.

\title{
COOPERATION WHILE STRATEGY UNDER DIFFERENT THEORETI- CAL APPROACHES
}

\begin{abstract}
This paper aims to identify the central focus, the main used arguments, the theoretical bases and the central conclusions about interorganizational cooperation in the end of 1990. The research is guided through the revision of the specialized literature and it concludes that the scientific works on the field of cooperation strategy follow three types of approaches: investigations concerning the relationship between the use of cooperative strategies and the performance of the firms or as source of a competitive advantage; studies on the process of formation of the interorganizational cooperative arrangements; and the construction of models and frameworks emphasizing the aspects under the interfirm relationships. In this sense this paper also identify the theoretical approaches underlying the works on this field of the strategic administration and interorganizational cooperative relationships.
\end{abstract}

Keywords: Interorganizational relationships. Strategies. Cooperation. 


\section{REFERÊNCIAS}

AFUAH, A. How much do your co-opetitors' capabilities matter in the face of technological change? Strategic Management Journal, v. 21, n. 3, p. 397-404, 2000.

AHUJA, G. The duality of collaboration: inducements and opportunities in the formation of Interfirm Linkages. Strategic Management Journal, v. 21, n. 3, p. 317-343, 2000.

ANAND, B. N.; KHANNA, T. Do firms learn to create value? The case of alliances. Strategic Management Journal, v. 21, n. 3, p. 295-315, 2000.

BARKEMA, H. G.; SHENKAR, O.; VERMEULEN, F.; BELL. J. H. J. Working abroad, working with others: how firms learn to operate international joint ventures. Academy of Management Journal, v. 40, n. 2, p.426-442, 1997.

BAUM, J. A. C.; CALABRESE, T.; SILVERMAN, B. S. Don't Go It Alone: Alliance Network Composition and Startups' Performance in Canadian Biotechnology. Strategic Management Journal, v. 21, n. 3, p. 267-294, 2000.

BHAPPU, A. D. The Japanese family: an institutional logic for Japanese corporate networks and Japanese management. The Academy of Management Review, v. 25, n. 2, p. 409-415, 2000.

BOWERSOX D. J.; CLOSS, D. J. Logistical Management: the Integrated Supply Chain Process. NY: McGraw-Hill, 1996.

BRANDENBURG, A.; NALEBUFF, B. Co-opetition. New York: Doubleday, 1996.

BROWNING, L. D.; BEYER, J. M.; SHETLER, J. C. Building cooperation in a competitive industry: SEMATECH and the semiconductor industry. Academy of Management Journal, v. 38, n. 1, p. 113-151, 1995.

CHILD, J.; FAULKNER, D. Strategies of co-operation: managing alliances, network, and joint ventures. Oxford: Oxford University Press, 1998.

CHUNG, S.; SINGH, H.; LEE, K. Complementarity, status similarity and social capital as drivers of alliance formation. Strategic Management Journal, v. 21, n. 1, p. 1-22, 2000. 
Cooperação como estratégia segundo diferentes perspectivas teóricas

COMBS, J. G.; KETCHEN Jr, D. J. Explaining interfirm cooperation and performance: toward a reconciliation of predictions from the resource-based view and organizational economics. Strategic Management Journal, v. 20, n.9, p. 867-888, 1999.

COOPER, M. C.; LAMBERT, D. M.; PAGH, J.D. Supply Chain Management: more than a new name for logistics. The International Journal of Logistics Management, v.8, 1997.

CRAVENS, D.; PIERCY, N. Relationships Marketing and Colaborative Network in service organizations. International Journal of Services Industry Management, v.5, n.5, 1994.

DAS, S.; SEN, P. K.; SENGUPTA, S. Impact of strategic alliances on firm valuation. Academy of Management Journal. v. 41, n. 1, p. 27-43, 1998.

DAS, T. K; TENG, B. Between trust and control: Developing confidence in partner cooperation in alliances. The Academy of Management Review, v. 23, n. 3, p. 491-512, 1998.

DICKSON, P. H.; WEAVER, K. M. Environmental determinants and individuallevel moderators of alliance use. Academy of Management Journal, v. 40, n. 2, p. 404-425, 1997.

DOLLINGER, M. J.; GOLDEN, P. A.; SAXTON, T. The Effect of Reputation on the Decision to Joint Venture. Strategic Management Journal, v. 18, n. 2, p. 127-140, 1997.

DONEY, P. M.; CANNON, J. P.; MULLEN, M. R. Understanding the influence of national culture on the development of trust. The Academy of Management Review, v. 23, n. 3, p. 601-620, 1998.

DOZ, Y. L. The Evolution of Cooperation in Strategic Alliances: initial conditions or learning processes. Strategic Management Journal, v. 17, p. 55-84, 1996.

DYER, J. H. Specialized Supplier Networks as a Source of competitive advantage: evidence from the Auto Industry. Strategic Management Journal, v. 17, n. 4, p. 271-291, 1996. 
. Effective Interfirm Collaboration: how firms minimize transaction costs and maximize transaction value. Strategic Management Journal, v. 18, n. 7, p. 535-556, 1997.

GHOSHAL, S.; BARTLETT, C. A. The Multinational Corporation as an Interorganizational Network. The Academy of Management Review, v. 15, n. 4, p. 603-625, 1990.

GIL, A. C. Como elaborar projetos de pesquisa. 3. ed. São Paulo: Atlas, 1991.

GOES, J. B.; PARK, S. H. Interorganizational links and innovation: the case of hospital services. Academy of Management Journal, v. 40, n. 3, p. 673-696, 1997.

GRANDORI, A.; SODA, G., Inter-firm Netoworks: antecedents, mechanisms and forms. Organization Studies, 1995.

GRANT, R. M.; BADEN-FULLER, C. A. Knowledge-based theory of inter-firm collaboration. Academy of Management Journal. Discovery, p. 17-21, 1995.

GULATI, R. Does familiarity breed trust? The implications of repeated ties for contractual choice in alliances. Academy of Management Journal, v. 38, n. 1, p. 85-112, 1995.

. Alliances and Networks. Strategic Management Journal, v. 19, n. 4, p. 293-317, 1998.

Network Location and Learning: the influence of network resources and firm capabilities on alliance formation. Strategic Management Journal, v. 20, n. 5, p. 397-420, 1999.

GULATI, R.; NOHRIA, N.; ZAHEER, A. Strategic networks. Strategic Management Journal, v. 21, n. 3, p. 203-215, 2000.

HAGEDOORN, J.; SCHAKENRAAD, J. The effect of strategic technology alliances on company performance. Strategic Management Journal, v. 15, n. 4, p. 291, 1994.

HAGEN, J. M.; CHOE, S. Trust in Japanese interfirm relations: institutional sanctions matter. The Academy of Management Review, v. 23, n. 3, p. 589-600, 1998. 
Cooperação como estratégia segundo diferentes perspectivas teóricas

HEIDE, J. B.; MINER, A. S. The shadow of the future: effects of anticipated interaction and frequency of contact on buyer-seller cooperation. Academy of Management Journal, v. 35, n. 2, p. 265-291, 1992.

HILL, C. W. L. Cooperation, opportunism, and the invisible hand: implications for transaction cost theory. The Academy of Management Review, v. 15, n. 3, p. 500-513, 1990.

HOLM, D. B.; ERIKSSON, K; JOHANSON, J. Value Creation through Mutual Commitment to Business Network Relationships. Strategic Management Journal, v. 20, n. 5 , p. $467-486,1999$.

HUMAN, S. E.; PROVAN, K. G. An emergent theory of structure and outcomes in small-firm strategic manufacturing networks. Academy of Management Journal, v. 40, n. 2, p. 368-403, 1997.

INKPEN, A. C.; BEAMISH, P. W. Knowledge, bargaining power, and the instability of international joint ventures. The Academy of Management Review, v. 22, n. 1, p. 177-202, 1997.

JONES, C.; HESTERLY, W. S.; BORGATTI, S. P. A general theory of network governance: Exchange conditions and social mechanisms. The Academy of Management Review, v. 22, n. 4, p. 911-945, 1997.

KALE, P.; SINGH, H.; PERLMUTTER, H. Learning and Protection of Proprietary Assets in Strategic Alliances: Building Relational Capital. Strategic Management Journal, v. 21, n. 3, p. 217-237, 2000.

KHANNA, T.; GULATI, R.; NOHRIA, N. The dynamics of learning alliances: competition, cooperation and relative scope. Strategic Management Journal, v. 19, n. 3, p. 193-210, 1998.

KOGUT, B. The Network as Knowledge: generative rules and the emergence of structure. Strategic Management Journal, v. 21, n. 3, p. 405-425, 2000.

KOH, J.; VENKATRAMAN, N. Joint Venture formations and stock market reactions: an assessment in the Information Technology Sector. Academy of Management Journal, v. 34, n. 4, p. 869-892, 1991. 
LADO, A.; BOYD, N. G.; HANLON, S. C. Competition, cooperation, and the search for economic rents: a syncretic model. The Academy of Management Review, v. 22, n. 1, p. 110-141, 1997.

MADHAVAN, R.; PRESCOTT, J. E. Market value impact of joint ventures: the effect of industry information-processing load. Academy of Management Journal, v. 38, n. 3, p. 900-915, 1995.

MITCHELL, W.; SINGH, K. Survival of businesses using collaborative relationships to commercialize complex goods. Strategic Management Journal, v. 17, n. 3, p. 169-195, 1996.

MOHR, J.; SPEKMAN, R. Characteristics of partnership success: partnership attributes, communication behavior, and conflict resolution techniques. Strategic Management Journal, v. 15, n. 2, p. 135-152, 1994.

MORVAN, Y., Filière de Production: fondementes d'Economie Industrielle. Paris: Economica, 1985.

MOWERY, D. C.; OXLEY, J. E.; SILVERMAN, B. S. Strategic alliances and interfirm knowledge transfer. Strategic Management Journal, v. 17, Special Issue, p.77-91, 1996.

NIELSEN, R. P. Cooperative Strategy. Strategic Management Journal, v. 9, n. 4, p. 475-492, 1988.

NOOTEBOOM, B.; BERGER, H.; NOORDERHAVEN, N. G. Effects of trust and governance on relational risk in alliances. Academy of Management Journal, v. 40, n. 2, p. 308-338, 1997.

OLIVER, C. Determinants of Interorganizational Relationships: integration and future directions. The Academy of Management Review, v. 15, n. 2, p. 241-265, 1990.

OSBORN, R. N.; HAGEDOORN, J. The institutionalization and evolutionary dynamics of interorganizational alliances and networks. Academy of Management Journal, v. 40, n. 2, p. 261-278, 1997.

PARK, S. H.; UNGSON, G. R. The effect of national culture, organizational complementarity, and economic motivation on joint venture dissolution. Academy of Management Journal, v. 40, n. 2, p. 279-307, 1997. 
PARKHE, A. Strategic alliance structuring: a game theoretic and transaction cost examination of interfirm cooperation. Academy of Management Journal, v. 36, n. 4, p. 794-829, 1993.

PEARCE, R. J. Toward understanding joint venture performance and survival: a bargaining and influence approach to transaction cost theory. The Academy of Management Review, v. 22, n. 1, p. 203-225, 1997

PERROW, C. Small Firm Networks. In: Nohria, N., Eclles, R., Networks and Organizations: structure, form and action. Boston: Harvard Business School Press, 1992.

RING, P. S.; VAN DE VEN, A. H. Structuring cooperative relationships between organizations. Strategic Management Journal, v. 13, n. 7, p. 483-498, 1992.

. Developmental processes of cooperative interorganizational relationships. The Academy of Management Review, v. 19, n. 1, p. 90-118, 1994.

SAKAKIBARA, M. Heterogeneity of firm capabilities and cooperative research and development: an empirical examination of motives. Strategic Management Journal, v. 18 Supplement, p. 143-164, 1997.

SAXTON, T. The effects of partner and relationship characteristics on alliance outcomes. Academy of Management Journal, v. 40, n. 2, p. 443-460, 1997.

SIMONIN, B. L. Ambiguity and the process of knowledge transfer in strategic alliances. Strategic Management Journal, v. 20, n. 7, p 595-623, 1999.

SINGH, K. The impact of technological complexity and interfirm cooperation on business survival. Academy of Management Journal, v. 40, n. 2, p. 339-367, 1997.

STEENSMA, H. K et al. The influence of national culture on the formation of technology alliances by entrepreneurial firms. Academy of Management Journal, v. 43, n. 5, p. 951-973, 2000.

STUART, T. E. Interorganizational alliances and the performance of firms: a study of growth and innovation rates in a high-technology industry. Strategic Management Journal, v. 21, n. 8, p. 791-811, 2000.

TSAI, W. Social Capital, strategic relatedness and the formation of interorganizational Linkages. Strategic Management Journal, v. 21, n. 9, p. 925-939, 2000. 